\title{
Reanastomosis Tubárica y otras técnicas Microquirúrgicas en Infertilidad
}

\author{
Dres. Gustavo Gómez Tabares*, Silvio Heriberto Estela González**
}

La esterilización quirúrgica en la pasada década se ha incorporado como método masivo de control de la fertilidad. Hasta 1985 se habían esterilizado por esta técnica, 6 millones de mujeres norteamericanas por debajo de 30 años de edad. En nuestro medio el $65 \%$ de las mujeres en edad reproductiva planifican; de estas el 18\% lo hacen por métodos quirúrgicos, $16 \%$ usan anticonceptivos orales y el $11 \%$ utilizan el DIU $(1,2,3)$. En Norteamérica se ha estimado que alrededor del $1 \%$ de estas pacientes, buscan reanastomosis -500.000 anualmente - . La razón usual para solicitud de reanastomosis tubárica es la separación conyugal y una nueva unión $(4,5)$. En la actualidad la esterilización quirúrgica es el método más usado en mujeres por encima de 30 años de edad (2). La reanastomosis tubárica post ligadura se ha incrementado al $49 \%$ de la microcirugía por infertilidad que realizamos en la Clínica de Infertilidad y Endocrinología del Departamento de Ginecología del Hospital Universitario del Valle. El 51\% restante, lo ocupan los demás procedimientos microquirúrgicos tubáricos

* Profesor Asociado

Departamento de Obstetricia y Ginecología

Facultad de Salud

Universidad del Valle

Director Clínica Endocrinología e Infertilidad Hospital Universitario del Valle

** Residente Obstetricia y Ginecología

Universidad del Cauca

Rotante por la Clínica de Endocrinología

e Infertilidad - 1988

Departamento de Obstetricia y Ginecología

Universidad del Valle dirigidos a corregir la infertilidad de origen tuboperitoneal.

\section{FISIOLOGIA DE LA TROMPA DE FALOPIO}

La reconstrucción tubárica debe contemplar, además de la canalización y permeabilización tubárica, entender hasta lo posible que, la función de la trompa, no es el de servir únicamente como vía de comunicación entre el ovario y el útero, sino que, la labor se complementa necesaria y de manera imprescindible, con una función de transporte, nutrición y protección indispensable para el éxito de su acción en reproducción, fecundación y transporte del oocito fertilizado.

Fisiológicamente hablando, los elementos importantes en la función tubárica son la captura y el transporte del oocito y el oocito fecundado, cuando esto ocurre, y el desplazamiento del espermatozoide.

Tradicionalmente se ha considerado que la fimbria en estrecho contacto con el ovario, "barre" y "abraza" el folículo preovulatorio para capturar el oocito con su células granulosas acompañantes. Se han demostrado movimientos específicos tubofímbricos en la época preovulatoria (6). La relación anatómica de la fimbria con el ovario, destaca la presencia de la porción fímbrica, unida al ovario, con el ligamento tubo ovárico, que fija la trompa y le permite movimientos a la fimbria (7). Esta porción de la fimbria es de suma importancia y la conservación de la mucosa, junto con una superficie adecuada, son esenciales para que su función se realice adecuadamente (8).

El incremento de la investigación, con la observación directa del fenómeno de ruptura folicular 
y expulsión del oocito, ha aumentado los convencidos, entre los cuales nos encontramos, de que la mayoría de las veces la captura del oocito se realiza en el fondo de saco de Douglas. El área de la fimbria realza su importancia en este caso. La explicación de embarazos con la existencia de ovario y trompa contralateral únicos, están a favor de ese concepto. En el Rhesus se han encontrado hasta el $70 \%$ de los occitos en el saco de Douglas, lo que implica que la ovulación ha ocurrido hacia cavidad, o que han fallado los métodos de captura (9). El contacto inicial del oocito con su cumulus ooforus, se hace con la mucosa de la fimbria, adhiriéndose a ella. Posteriormente los movimientos de la cilia con dirección al útero, participarán activamente en el mecanismo de transporte al tercio distal de la ampolla, donde se efectuará la fecundación. La indispensabilidad y eficiencia de la fimbria en la captura del oocito, se ha estudiado amplia y profundamente especialmente en conejos. Se ha demostrado relación directa entre el grado de deciliación de la fimbria y la eficiencia en la captura del oocito (10). La extirpación experimental de la fimbria en el conejo, ha reducido la captura del oocito, de un $80 \%$ en los controles, al $63 \%$ en el grupo estudio (11). Sin embargo el movimiento de cúmulos-oocito en la mucosa de la neostomía fue normal en la mayoría de los casos. En estudio similar, pero con extirpación más amplia, hasta la mitad de la ampolla, el porcentaje de captura del oocito fue $29 \%$ en las neostomías versus $77 \%$ en los controles (12). Resección de la fimbria con más del $50 \%$ de la ampolla, reduce a cero la captura del cúmulus-oocito (13). Sin embargo, a pesar de la similitud en algunos aspectos de la trompa del conejo y el humano, hay diferencias que pueden ser importantes para equiparar estos hallazgos en las dos especies (14). Lo que nos induce a pensar que aunque no es absolutamente indispensable la presencia de fimbria natural, si lo debe ser lo más similar a ella en la reconstrucción. Una neostomía muy proximal en la ampolla no es, ni mucho menos, de pronóstico favorable en restablecer la fertilidad.

La composición de la mucosa de la fimbria y la ampolla es similar, con células ciliadas y secretoras. Las primeras predominan, sobre las segundas. Estas a su vez disminuyen severamente en la primera porción de la ampolla (15). El transporte del oocito en la ampolla, se realiza por medio del movimiento de las cilias, las corrientes intratubáricas del líquido tubárico. El movimiento de las cilias con dirección uterina se ha documentado suficientemente (16). Las contracciones musculares de la trompa se han medido, registrando la actividad mioeléctrica, por medio de electrodos de succión adheridos al tejido muscular (17). Ambos sistemas responden con variaciones en intensidad y frecuencia a los cambios hormonales del ciclo (18). In vitro, porciones de oviductos, muestran transferencia de microesferas, con dirección uterina (19). La actividad de las cilias bajo concentración aumentada de estrógenos, como sucede en la época preovulatoria, es más activa (18). La implantación invertida de la porción ampular, en forma experimental, en el conejo, bloquea la progresión del oocito hacia el útero, muy probablemente por la persistencia del movimiento ciliar hacia el útero (20). Sin embargo, la presencia de embarazos y el transporte demostrado del oocito en ausencia de movimiento ciliar de la mucosa, enfatiza la participación de los otros mecanismos de transporte de la trompa. El oocito permanece aproximadamente 72 horas en la ampolla y una vez fecundado hace una pausa en lo que se ha llamado el esfínter o unión ístmico-ampular, que no es un sitio anatómicamente definido, aunque funcionalmente si lo es (21). Experimentalmente en animales, la resección de este sitio no afecta de manera importante la fertilidad. En este sitio se ha descrito un marcapaso de conducción eléctrica de la trompa (22).

En la transición de la ampolla hacia el istmo, se reduce marcadamente la luz de la trompa. Los pliegues mayores de la mucosa tubárica de la ampolla se reducen a tres o cuatro pliegues menores ístmicos, redondeados de 200 a 400 micras de altura. El componente muscular es más prominente y fuerte. Las células ciliadas disminuyen y aumentan las secretoras a un $80 \%$ de todas las que componen la mucosa (23). Estas células responden de una manera directa a las concentraciones hormonales circulantes. La acción de los estrógenos incrementan la producción de secreciones, que en la época preovulatoria inmediata es tan abundante que oculta la acción de las células ciliadas y sirve de medio para que el espermatozoide encuentre la resistencia adecuada a los movimientos de su flagelo y se desplace hacia la ampolla (15). Una vez ocurre la ovulación, la aparición de la progesterona, junto con la disminución de estrógenos, aclara la luz tubárica de secreciones y la acción del movimiento ciliar hacia el útero, proporciona el movimiento del embrión hacia el sitio de implantación (15). La acción de las paredes musculares, parece tiene más importancia en la región ístmica, para el transporte del embrión. Se ha demostrado actividad eléctrica a partir de marcapaso de la unión ístmico-ampular, hacia otro en la unión ístmico-cornual (24). El desplazamiento del cúmulus-oocito o del embrión se 
haría por contracción de la musculatura tubárica, en el sentido de alejarse de una zona activa y entrar en una inactiva, eléctricamente hablando (24). La estancia y el paso del embrión por el istmo, es relativamente rápido.

\section{TECNICAS MICROQUIRURGICAS}

La innovación en las técnicas microquirúrgicas en infertilidad reside básicamente, no en la técnica en sí, sino en la evolución en la magnificación del campo, el uso de microinstrumental, acciones farmacológicas y terapéuticas para prevenir la formación de adherencias, y la actitud mental y entrenamiento del especialista en infertilidad, que debe estar enfocado no sólo en el manejo quirúrgico de la paciente, sino con una visión integral de la pareja con todos los factores involucrados en el estudio de infertilidad. Las técnicas quirúrgicas propiamente dichas se pueden agrupar en: Reanastomosis Tubárica, Salpingoneostomía o salpingostomía, Fim. brioplastia y Liberación de Adherencias.

\section{Reanastomosis Tubárica}

Las primeras operaciones para corregir trompas ocluidas como salpingoneostomía o anastomosis fueron descritas por Siegler (25). En 1908 se informa la primera reanastomosis término-terminal, en oviducto de gallina (26). En 1913 se reportan las primeras reanastomosis ístmico-ístmico en tres mujeres, en las cuales se deja una guía de catgut durante 20 días (27).

Similar a todas las intervenciones microquirúrgicas, actualmente se utilizan los métodos contemporáneos como: uso de microscopio operatorio, técnica atraumática, instrumental microquirúrgico de ginecología, irrigación en el sitio operatorio, aproximación estable de los segmentos tubáricos y el uso de suturas con agujas finas y material no reactivo (28).

Las anastomosis se clasifican: intramural-ístmica, intramural-ampular, ístmica-ístmica, ístmicaampular y ampulo-ampular.

Los pasos quirúrgicos de la reanastomosis propiamente dicha incluye: exposición del campo, exposición del útero, levantándolo y "empacando" con compresas húmedas en el fondo de saco de Douglas para sostenerlo alto en el campo; identificación de los segmentos a anastomosar, así: proximal, instilando colorante a través de la cavidad uterina ya sea por inyección retrógrada con un equipo de microgoteo, o por inserción de una sonda de folley o similar en la cavidad uterina; distalmente se explora instilando colorante de manera retrógrada a través de la fimbria, utilizando: un cateter R33 manipulando la trompa delicadamente con una pinza de trompas.

El segmento obstruido se secciona con electrocauterio, haciéndolo estrictamente en la zona obstruída. Una vez identificados los dos extremos, se despega en cada uno $3 \mathrm{~mm}$ de serosa, de la capa muscular; esto evitará que la unión de la anastomosis se acode al cierre de la serosa. Se abre la luz de la trompa, cortando una pequeña rodaja con instrumental cortante. Se coloca un punto de aproximación y disminución de tensión, simétrico, inmediatamente por debajo de la musculatura de ambos extremos con prolene 6 ceros. Las capas musculares se suturan con nylón 8 ceros, teniendo cuidado de anudar hacia afuera y de no entrar, a la luz de la trompa. En la región intramural e ístmica se necesitan 4 puntos. En la ampular pueden ser 2 ó 3 más. La serosa se sutura con puntos invaginantes separados de prolene 7-0 aguja redonda. Diferente a las otras técnicas microquirúrgicas, no se usan esteroides sistémicos, pero sí antibióticos profilácticos.

\section{Salpingoneostomía - Salpingostomía}

Desde 1932 Sovak y Holden publicaron y describieron la técnica de eversión de los bordes de una trompa obstruida, con el fin de permeabilizarla (29). Los resultados, con consecución de embarazos, fueron pobres. Desde entonces se han publicado numerosos trabajos al respecto $(30,31,32,33$, $34,35)$.

La clasificación de la obstrucción distal de la trompa no es unánime. En el IX Congreso Mundial de Fertilidad y Esterilidad en 1977 se clasificó como: Salpingoneostomía - Salpingostomía - a) terminal; b) ámpulo-medial, y, c) ístmico-incluyendo salpingostomía líneal-. Algunos han hecho objeto de controversia el considerar si cuando se encuentra una fimbria escondida - "criptofimbria", se considera salpingostomía o fimbrioplastia el tipo de cirugía a realizar. Nosotros aplicamos el término de fimbrioplastia cuando la obstrucción es parcial. $\mathrm{Si}$ es total, la denominamos salpingoneostomía.

La técnica quirúrgica clásica, convencional y contemporánea en salpingoneostomía, fue descri. ta por Kistner en 1975 (36). Bajo visión microscópica, previa instilación de colorante a través del útero para patentizar la obstrucción distal, se iden- 
tifican las zonas avasculares, por las cuales se hace incisión con electrocauterio. Se evierten los colgajos resultantes y se fijan a la serosa con nylón 8-0. Cuando la pared de la trompa está muy engrosada, los puntos iniciados en el borde de la mucosa, deben invaginar la pared muscular. Como en todas las demás técnicas microquirúrgicas, la hemostasia debe ser de especial cuidado. Las técnicas microquirúrgicas, enfatizan en el manejo delicado de los tejidos, el uso de instrumental de puntas finas y minimizando la tensión en las líneas de sutura.

\section{Fimbrioplastia}

Se entiende que la trompa es, aunque parcialmente, permeable. La clasificación tiene en cuenta el estado de la fimbria per se y el grado de dificultad al paso del medio de contraste. En la primera situación hablamos de fimbria "aglutinada" o en "hisopo", con ausencia de fimbria ovárica o "fimbria ovárica escasa'. Con relación a la dificultad para el paso del medio de contraste, la denominamos FIMOSIS, que clasificamos como: leve, moderada y severa. Las dos últimas son de manejo quirúrgico. La fimosis moderada implica dificultad para el paso del medio de contraste, con dilatación persistente hasta el segmento proximal de la ampolla, que subsiste después de que el colorante ha salido a cavidad peritoneal. En la fimosis severa el paso del colorante es más difícil, a veces salen unas gotas después de que la trompa se dilata hasta cualquiera de las porciones del istmo. Habitualmente se encuentra una construcción o "cincha", en el infundibulo, inmediatamente antes de iniciarse la fimbria (Fig. 1). Generalmente cualquier grado de

FIGURA 1

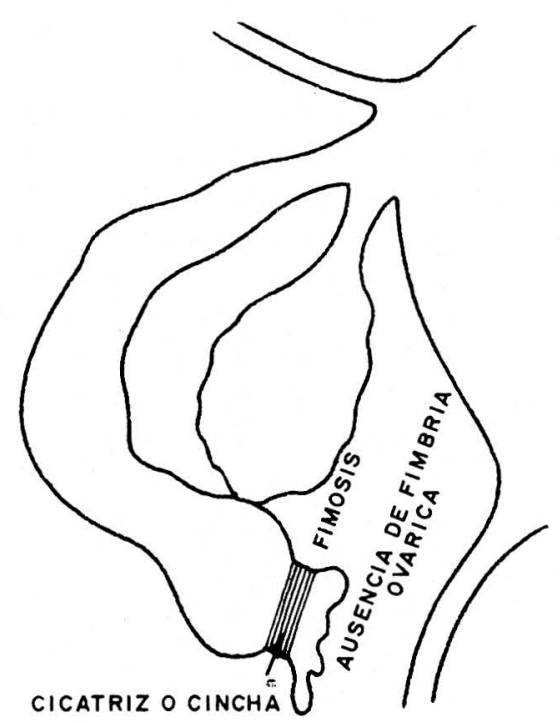

fimosis, está acompañado de fimbrias "aglutinadas", es decir, que no se extienden, disminuyendo considerablemente su área o superficie (Fig. 2).

FIGURA 2

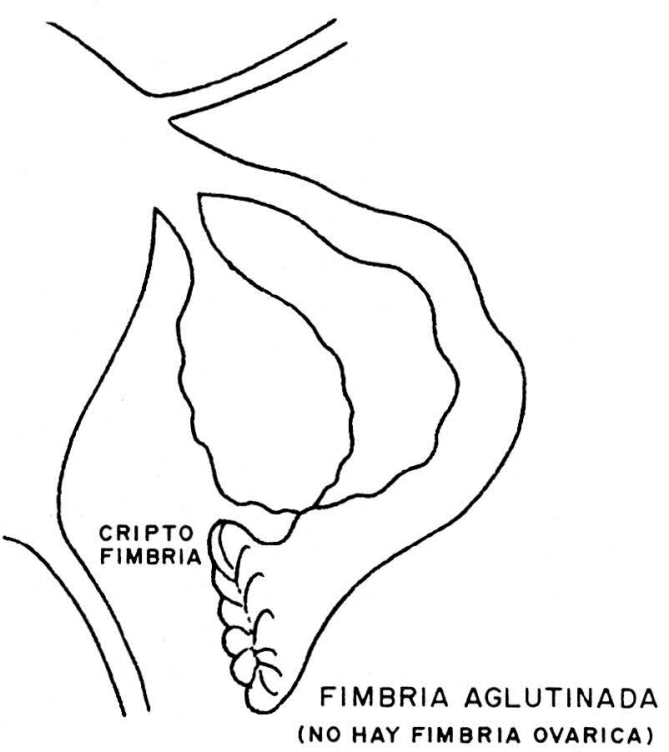

La fimbria en "hisopo", no muestra engrosamiento, pero la trompa se continúa con ella en forma tubular, con los pliegues separados entre sí, dejando espacios entre ellos, también con disminución del área de la fimbria y ausencia de fimbria ovárica (Fig. 3). El manejo quirúrgico se orienta a la normalización del calibre de la luz de la trompa, a la creación o reconstrucción de la fimbria ovárica y a aumentar el área de la misma.

\section{FIGURA 3}

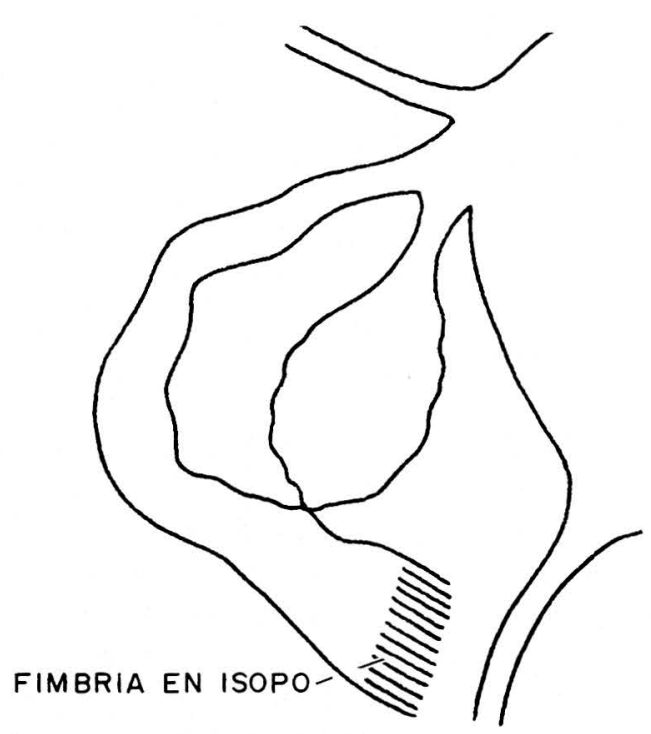

(NO HAY FIMBRIA OVARICA 
Una vez identificado el tipo de lesión, con electrocauterio se seccionan las adherencias entre los pliegues de la fimbria. Para crear la fimbria ovárica y/o aumentar la superficie de la misma, se hace una incisión con electrocauterio, antimesentérica, cuidando la hemostasia e irrigando permanentemente con la solución de irrigación, solución de Hartman 1000 cc más 100 mgrs de Hidrocortizona. Una vez hecho lo anterior se fijan los bordes de la fimbria ampula escindida, a la serosa de la trompa en una dirección de eversión. Hay que tener en cuenta que el diámetro de la luz de la trompa, disminuye a medida que se asciende hacia el útero, por lo tanto con esta incisión lo que se logra es disponer de mayor superficie de mucosa ampular, que va a funcionar como fimbria ovárica y no de aumentar el orificio tubárico.

\section{Liberación de Adherencias}

No podemos hablar de una técnica específica, cuando de liberación de adherencias se trata. La clasificación de las adherencias, ha dificultado la unificación de los resultados con las diferentes técnicas. Estas técnicas se refieren más a la prevención farmacológica de la producción o reproducción de adherencias.

Las adherencias “blandas", laxas o transparentes son de buen pronóstico quirúrgico, no sangran y se pueden liberar con instrumentos cortante romo. Tienen especial importancia las que envuelven el ovario, formando lo que llamamos un "pterigio" ovárico (Fig. 4). Pueden ser "gruesas", en cuyo caso, sangran y dejan superficies despulidas cuando se resecan. Es una de las situaciones en que es verdaderamente indicado el uso del laser para vaporizarlas.

La técnica microquirúrgica, desde la incisión en piel, está orientada a la prevención de la formación de adherencias. La hemostasia cuidadosa, el manejo delicado de los tejidos, el uso de magnificación y la utilización de fino material de sutura, hacen parte de la técnica microquirúrgica en general. Las adherencias se liberan con electrocauterio unipolar. Las adherencias gruesas pegadas al útero que no interfieren con la trompa y el ovario, es preferible dejarlas en su sitio para no producir zonas despulidas en las serosas, que son más difíciles de manejar. La peritonización de estas zonas, cuando se producen por separación de dos órganos contiguos deben ser peritonizados o, si es necesario, colocarles un injerto de peritoneo parietal.

\section{FIGURA 4}

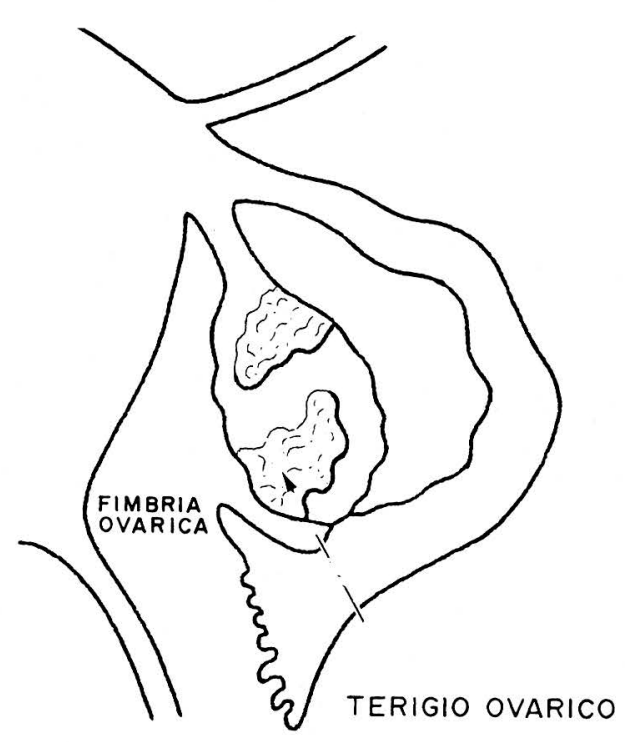

La prevención de adherencias es el reto mayor de la microcirugía en particular y de la cirugía pélvica en general. Con el fin de reducir este problema se han utilizado en el período perioperatorio, una amplia gama de medicamentos. Se han usado esteroides, antibióticos profilácticos, dextranes, y otras sustancias (37). Recientemente se esta orientando la atención a fármacos antiinflamatorios no esteroideos, esto, debido a que la formación de adherencias, en su proceso patogénico, implica una respuesta inflamatoria primaria en el área expuesta al insulto quirúrgico $(38,39)$. Después del trauma o la injuria, se produce infiltración leucocitaria en el área dañada.

Sustancias liberadas por estas células, atraen a otras, incluyendo plaquetas, con formación de fibrina. Lo anterior explica el uso de heparina local, intraoperatoria. La consecuente producción de prostaglandinas, mediadores de la respuesta inflamatoria del tejido lesionado es el paso siguiente. Los antiinflamatorios no esteroides inhiben la enzima ciclo-oxigenasa, inhibiendo la síntesis de prostaglandinas (40). El resultado con el uso de estos medicamentos no es aún concluyente (41, $42,43)$.

El esquema farmacológico para la prevención de adherencias en nuestra experiencia, es a base de esteroides y antibióticos profilácticos. $20 \mathrm{mgs}$ de dexametazona im. o ev. la noche anterior, la mañana y la noche de la cirugía, es la dosis inicial; el segundo día 1 mgr de dexametazona cada seis horas y posteriormente, durante cinco días $0.75 \mathrm{mgs}$ de dexametazona oral cuatro veces por día. 
La solución de irrigación intraoperatoria se prepara con 1 litro de solución de Hartman más $100 \mathrm{mgs}$ de hidrocortizona.

Al terminar la cirugía se deja en cavidad 20 mgs de dexametazona en cavidad en un vehículo que hace su absorción lenta y permite una acción local más prolongada. El uso de heparina se reserva para aquellos casos donde las superficies cruentas son importantes. El uso de antibióticos profilácticos es a base de 4 dosis de 1 gramo de una cefalosporina. Una dosis preoperatoria y post-operatoria inmediata; posteriormente dos dosis con intervalo de cuatro horas, dosis total.

\section{PACIENTES. METODOS.}

Las pacientes que consultan por infertilidad a la Clínica de Infertilidad y Endocrinología del HUV, son evaluadas en pareja. El estudio inicial incluye espermograma -único si es normal, repetidos una o dos veces, si es patológico-, CTB, Test post-cóito y laparoscopia diagnóstica. En el acto de la laparoscopia, programada para la segunda mitad del ciclo, se obtiene una biopsia de endometrio. Si en la laparoscopia encuentra patología tubo-uterina-peritoneal, se realiza una histerosalpingografía, antes de programarse la paciente para microcirugía. En el caso particular de las pacientes que buscan recanalización post-ligadura, prescindimos de la histerosalpingografía posterior, pues consideramos que la información sobre êl segmento distal, estado y conservación de la fimbria no se obtiene con el procedimiento, aunque colocamos especial cuidado en la permeabilidad del segmento proximal durante la laparoscopia, si no se puede identificar con certeza, recomendamos el procedimiento radiológico previo a la cirugía.

Se analizaron las últimas 90 microcirugías derivadas directamente de la Clínica de Infertilidad del HUV. El tiempo de seguimiento mínimo, fue de cuatro meses. No volvieron, o lo hicieron de una manera muy irregular 31 de las pacientes, que implica deserción del $34 \%$. No se tienen datos consistentes de la evolución de ellas.

Además del factor tuboperitoneal comprometido, de las 59 pacientes restantes, había compromiso en $8(13.5 \%)$, del factor cervical; 3 pacientes presentaban anovulación $(5.0 \%)$ y 2 tenían compromiso del factor masculino $(3.3 \%)$.

Se realizaron 40 reanastomosis de las cuales no hay seguimiento en $11 ; 31$ fimbrioplastias sin seguimiento en 14; salpingostomía en 9 , sin seguimiento en 2; y liberación de adherencias en 10, sin control posterior en 4 (cuadro 1).

\section{CUADRO No. 1 \\ TIPOS DE MICROCIRUGIAS REALIZADAS SEGUIMIENTO 1988}

\begin{tabular}{|lrrrrrr|}
\hline & & & & Sin & & Con \\
& $\%$ & No. & $\%$ & Segui. & $\%$ & Segui. \\
\hline Reanastomosis & 44.4 & 40 & 27.5 & 11 & 72.5 & 29 \\
Fimbrioplastia & 34.4 & 31 & 45.1 & 14 & 54.8 & 17 \\
Salpingoneostomia & 10.0 & 9 & 22.2 & 2 & 77.7 & 7 \\
Liberación Adherenc. & 11.1 & 10 & 40.0 & 4 & 60.0 & 6 \\
\hline \multicolumn{1}{|c}{ TOTAL } & 100.0 & 90 & & 31 & & 59 \\
\hline
\end{tabular}

Los resultados se contabilizan excluyendo las pacientes sin seguimiento.

Para efectos de descripción y resultados se dividieron las cirugías en, únicas, sin otra cirugía sobreagregada, y múltiples, aquellas en las que se había realizado más de un procedimiento o tipo de cirugía. Se contabilizaron: Reanastomosis 27; más liberación de adherencias 1; más fimbrioplastia 1. Fimbrioplastias 5; más liberación de adherencias 11 . Salpingoneostomías 1; más liberación de adherencias 8. Liberación de adherencias 5. Para un total de 59 pacientes.

Los porcentajes de embarazos intrauterinos a término, fueron: reanastomosis $51.8 \%$; fimbrioplastia $41.2 \%$; liberación de adherencias $16.6 \%$ y salpingoneostomía, no embarazos.

Un embarazo extrauterino se presentó, antes del año post-operatorio en las pacientes con reanastomosis $-3.4 \%$ de incidencia en este grupo y $1.6 \%$ en todos los casos-.

La totalidad de los embarazos en las fimbrioplastias se logro en los primeros cinco meses postoperatorios. El $84.6 \%$ de los embarazos en la reanastomosis se alcanzó en los primeros 12 meses. Uno de ellos fue un embarazo extrauterino a los 7 meses de una reanastomosis ístmica ampular bilateral. Tres de los embarazos terminaron por cesárea debido a indicaciones obstétricas.

\section{COMENTARIOS}

Paradójicamente, debido al auge de la ligadura de trompas, que la convierte en el método más 
usado, en planificación familiar, la reanastomosis tubárica se ha convertido en la reina de los procedimientos microquirúrgicos en infertilidad.

Primero por las buenas tasas de embarazos logrados con el procedimiento, y porque es el tipo de cirugía en que se pone en juego en forma integral, el concepto de microcirugía, que incluye adiestramiento adecuado del cirujano. Este debe tener una mentalidad orientada hacia el manejo conservador y delicado de los órganos reproductivos y es imperativo que conozca a fondo los mecanismos involucrados en el proceso reproductivo. E1 conocimiento de la anatomía y fisiología reproductiva y el entrenamiento quịuruico, son básicos para el éxito, el cual se mide por la tasa de embarazos conseguidos (45). E1 uso de microscopio operatorio, su manejo adecuado, para obtener libertad operatoria y comodidad y la utilización de instrumental adecuado es el complemento del cirujano. Instrumental de longitud adecuada (15 - $18 \mathrm{cms}$ ) con puntas finas de agarre perfecto y suturas con agujas atraumáticas y material que produzca poca reacción inflamatoria. Detalles como una mesa operatoria adecuada que permita al cirujano introducir sus piernas debajo, y manipular cauterio y zoom del microscopio, dan comodidad y disminuyen el cansancio y la tensión, lo que se traduce en pulso firme.

La creciente utilización de la ligadura tubárica, con el dramático aumento en separaciones y divorcios, unidos a la publicidad y la cada vez más creciente información sobre la reversibilidad del método, ha llevado al concepto de la ligadura como método no tan definitivo y que puede utilizarse durante cinco o más años - no mayor de ocho, por la disminución de las tasas de embarazo, a partir de entonces- y luego someterse a la recanalización. Algunas estadísticas muestran que el $1 \%$ de mujeres buscan este tipo de cirugía (46). La cifra, es seguro, se incrementará sostenidamente en el futuro.

Siegler y col, analizaron hasta 1985, 767 operaciones de recanalización y el porcentaje de nacidos vivos osciló entre el $47 \%$ y $78.8 \%$ y la incidencia de embarazo tubárico fue entre el $1.7 \%$ y $6.5 \%$ (45). En grandes series individuales - mayor de 50 reanastomosis- los embarazos obtenidos oscilan entre $60.4 \%$ y $82.5 \%$, con embarazos a término entre $47 \%-78 \%$ y una incidencia de embarazos extrauterinos entre $1.7 \%$ y $15.0 \%$ (46). En series menores - de 25 a 49 casos las tasas de embarazo fluctúan entre $39 \%$ y $60 \%$ con embarazos intrauterinos entre $25 \%$ y $57 \%$ y ectópicos entre $3.2 \%$ y $15 \%$
(46), lo que parece poner de manifiesto que la experiencia del equipo quirúrgico, supuesta por el mayor número de casos, es importante en los resultados. La incidencia de abandono en nuestro medio es alto, 11 de 40, de las cuales no conocemos su resultado. Obviado lo anterior la tasa de embarazos es del $51.8 \%$. Si consideramos que dos pacientes necesitaron otra cirugía concomitante, fimbrioplastia y liberación de adherencias, podemos mostrar una tasa de embarazos en reanastomosis, del $55.5 \%$ (cuadro 2). Con relación al sitio de la reanastomo-

CUADRO No. 2

REANASTOMOSIS EMBARAZOS A TERMINO 1988

\begin{tabular}{|lrrr|}
\hline & No. & Emb. & $\%$ \\
\hline Reanastomosis Post-Ligadura & 25 & 14 & 56 \\
Reanastomosis por Obstrucción & 2 & 1 & 50 \\
Reanastomosis+ Lib. Adherencias & 1 & 0 & 0 \\
Reanastomosis+ Fimbrioplastia & 1 & 0 & 0 \\
\hline \multicolumn{1}{|c}{ TOTAL } & 29 & 15 & 51.8 \\
\hline
\end{tabular}

sis la ístmica-ístmica es la que muestra mejores resultados, seguida de la ampulo-ampular y la ístmica temprana y el cuerno (cuadro 3). El tiempo qui-

CUADRO No. 3

PORCENTAJE NETO REANASTOMOSIS Y RESULTADO COMO EMBARAZO 1988

\begin{tabular}{|lrrrrrrrr|}
\hline & $\%$ & No. & $\begin{array}{l}\text { Emb. } \\
\text { Term }\end{array}$ & $\begin{array}{l}\text { Ecto- } \\
\text { pico }\end{array}$ & $\begin{array}{c}\% \\
\text { Abor- \% } \\
\text { tos }\end{array}$ \\
\hline Istmico-Cornual & 6.9 & 2 & 1 & 50 & - & - & - & \\
Istmico-Istmica & 20.68 & 6 & 6 & 100 & - & - & - & - \\
Istmico-Ampular & 59.72 & 15 & 6 & 40 & 1 & 14.3 & - & - \\
Ampulo-Ampular & 13.8 & 4 & 2 & 50 & - & - & - & - \\
Ampulo-Fímbrica & 6.9 & 2 & 0 & 0 & - & - &. & - \\
\hline TOTAL & 100.0 & 29 & & & & & \\
\hline
\end{tabular}

rúrgico en todos los casos de embarazo oscila entre 2:20 y 2:40 horas. Evidentemente, el diámetro similar de los extremos a reanastomosar, son muy importantes para el éxito de la cirugía y son las que proporcionan cirugías más rápidas, con menos trauma y exposición de los tejidos al medio ambiente y posibilidad de deshidratación. También hay diferencias en embarazos intrauterinos, de acuerdo con la técnica utilizada para la esteriliza- 
ción: clips $84 \%$; bandas $72 \%$; Pomeroy $59 \%$ y coagulación $41 \%$, de acuerdo con diferentes series en la literatura mundial $(45,47)$. Aún demostrada la permeabilidad tubárica postoperatoria. son diversos los factores que influyen en la consecusión de embarazos: la técnica quirúrgica de esterilización utilizada, -Pomeroy, bandas, clips, etc.-, la longitud de la trompa post-quirúrgica, (menor de $4 \mathrm{cms}$ de peor pronóstico) (47), reoclusión, otros factores de infertilidad, pobre técnica quirúrgica e inexperiencia. Por eso lo difícil de comparar entre las diferentes series de distintos autores. Se asume que después de una ligadura los segmentos intramurales permanecen normales, y la reanastomosis, siempre es posible. Sin embargo, algunos autores han encontrado endometriosis hidrosalpinx, salpingitis ístmica nodosa, fibrosis y salpingitis crónica, en ellos $(48,49,50)$.

La obstrucción distal patológica, definitivamente es de pronóstico más reservado. El resultado de la microcirugía del hidrosalpinx depende del tipo de hidrosalpinx. Se reconocen dos tipos: a) de pared gruesa - con infiltración inflamatoria de las paredes musculares, usualmente asociado a obstrucción distal con lumen que presenta el patrón trabecular del hidrosalpinx follicularis y en el cual la fimbria ovárica está dañada o destruida (51), y b) de pared delgada - que generalmente, contrario al anterior, tiene conservada la mucosa, aunque se ha descrito disminución hasta el $50 \%$ de las células ciliadas (52). El tamaño del hidrosalpinx, es reflejo del tiempo de duración de la lesión, e incide en la conservación de la mucosa. Existe correlación entre el tamaño del hidrosalpinx y el número de células ciliadas (53). Se puede especular que la disminución de las cilias, y trastornos de la funcionalidad de la capa muscular influyen en el pronóstico postquirúrgico, y por lo tanto en obtención de embara$\operatorname{zos}(54)$.

Los resultados descritos en la literatura con embarazo a término fluctúan entre $18 \%-29 \%$ con técnicas microquirúrgica y coadyuvante como esteroides e hidrotubaciones $(33,34,55)$. Los resultados con uso del laser, se sitúan entre el $7 \%$ - $24 \%$ de embarazos a término $(56,57)$.

El tipo de pacientes que recibimos y la inicial falta de selección de paciente, con relación a la severidad y duración de la lesión, explican los malos resultados en este tipo de cirugía, en nuestra experiencia.

La fimosis tubárica requiere un comentario más detallado.
Primero, porque generalmente la mucosa todavía está relativamente bien conservada, con los consiguientes beneficios que esto representa para el pronóstico. Segundo, porque hay que saber hacer el diagnóstico laparoscópico y aún radiológico de la lesión, lo que se consigue con observación detallada y experiencia metodizada. La fimbria aglutinada, que implica menor área o superficie de la mucosa y la pérdida de la fimbria ovárica, generalmente acompaña la lesión.

Consideramos como quirúrgica la fimosis moderada y severa la fimosis leve, es de más difícil diagnóstico, porque puede tratarse de una trompa normal. Hay que fijar muy detenidamente el estado de la fimbria -aglutinada, en hisopo, no fimbria ovárica-.

Los resultados de las series de fimbrioplastias muestran porcentajes de embarazos a término entre el 22 y 60. Las tasas de embarazo siempre fueron superiores, proporcional a la cantidad y calidad de fimbria presente $(58,59,60)$. En el medio hospitalario, la fimosis como patología única es baja.

Generalmente se acompaña de adherencias. No hay mucha diferencia entre una y otra en resultados (Cuadro 4 ). Combinación de técnicas quirúr-

CUADRO No. 4

FIMBRIOPLASTIAS EMBARAZOS A TERMINO 1988

\begin{tabular}{|cccr|}
\hline & No. & Emb. & $\%$ \\
\hline $\begin{array}{l}\text { Fimbrioplastias Puras } \\
\text { Fimbrioplastias + } \\
\text { Liberación Adherencias }\end{array}$ & 7 & 3 & 42.8 \\
\hline \multicolumn{1}{|c|}{ TOTAL } & 17 & 4 & 40.0 \\
\hline
\end{tabular}

\section{CUADRO No. 5 \\ EMBARAZOS SEGUN TECNICAS BASICAS DE MICROCIRUGIA 1988}

\begin{tabular}{|lrrr|}
\hline & No. & Emb. & $\%$ \\
\hline Reanastomosis & 29 & 15 & 51.8 \\
Fimbrioplastia & 17 & 7 & 41.2 \\
Liberación Adherenc. & 6 & 1 & 16.6 \\
Salpingoneostomía & 7 & 0 & 0 \\
\hline \multicolumn{1}{|c}{ TOTAL } & 59 & 23 & 38.9 \\
\hline
\end{tabular}


gicas, con mejor y más especializada ayuda tecnológica, el uso de fármacos para prevenir la formación de adherencias, la exclusión de pacientes de mal pronóstico ha aumentado levemente la incidencia de embarazos intrauterinos. Desafortunadamente es difícil estandarizar resultados, por la casi imposibilidad de agrupar las lesiones y también por la selección real del paciente, excluyendo los que pueden dañar las estadísticas, antes de la cirugía, o después de ella, cuando no se incluyen en las mismas.

A la luz de los datos disponibles actualmente, la cirugía con laser no proporciona mejores resultados, aunque algunos autores muestran embarazos más temprano después de la cirugía $(56,57,60)$.

\section{BIBLIOGRAFIA}

1. ORY H W., FORREST O J., LINCOLN R: Making choices: evaluating the health risks and benefits, of birth control methods. New York. The Alan Guttmacher Institue, 1983.

2. Center for Disease Control: Surgical Sterilization Surveillance, Tubal Sterilization Summary 1970 - 1975. Atlanta Georgia, July, 1979.

3. Encuesta de Dermografia y Salud en Colombia. 1986.

4. GOMEL V: Profile of women requesting reversal of sterilization. Fertil Steril 30: 39, 1978.

5. WISTON R M.L.: Why 103 women saked for reversal of sterilization. Br. Med. J. 2:305, 1977.

6. BLANDAU R J.: Observing ovulation and egg transport. In Methods in Mammalian embriology, Ed. J. C. Daniel Jr. San Francisco, W. H. Freeman, 1971, p.1

7. OKAMURA H., MORIKAWA H., OSHIMA M. MAN-i M., NISHIMURA T.: A morphologic study of mesotubarium ovarica in the human Obstet gynecol 49: 197, 1977.

8. OKAMURA H., MORIKAWA H., OSHIMA M. Man-i M., NISHIMURA T: A morphological and physiological study of mesotubarium in humans. Int J. Fertil 22: $179,1978$.

9. EDDY C. A., GARCIA R G., KRAEMER D.C., PAURSTEIN C J.: Detailed time course of ovum transport in the rhesus monkey (Macaca mulatta). Biol. Reprod. 13: 363, 1975.

10. ODOR DL. BLANDAU R J.: Egg transport over the fimbrial surface of the rabbit oviduct under experimental condictions. Fertil Steril 24: 292, 1973.

11. HALBERT SA PATTON DL: Ovum pick-up following fimbriectomy and infundibular salpingostomy in rabbits. J. Reprod. Med. 26: 299, 1981.

12. HALBERT SA., McCOMB PF., PATTON DL.: Function and structure of the rabbit oviduct following fimbriectomy. I Distal ampullary Salpingostomy. Fertil Steril 35: 349, 1981.

13. HALBERT SA., McCOMB PF : Function and structure of the rabbit oviduct following fimbriectomy. II Proximal ampullary salpingostomy. Fertil Steril 35: $355,1981$.
14. GADDUM-ROSSE P., BLANDAU R J.: In vitro studies on ciliary activity within the oviducts of the rabbit and pig. Am.J. Anat.136: 91, 1973.

15. JANSEN R P S.: Cycles changes in the human fallopian tube isthmus and their functional importance. Am.J. Obstet.Gynecol.136: 292, 1980.

16. HAFEZ ESE. Gamete transport. In: HAFEZ ESE, EVANS T N., eds. Human reproduction conception and contraception. New York: Harper and Row, 1973: 85.

17. HODGSON B J., TALO A., PAUERSTEIN C J.: Oviductal surrogate movement: inter-relation with muscular activity. Biol. Reprod. 16: 394, 1977.

18. TALO A. PULKKINEN M O.: Electrical activity in the human oviduct during menstrual cycle. Am J. Obstet Gynecol 142: 135, 1982.

19. GADDUM - ROSSE O, BLANDAU R J., THIERSCH $\mathrm{J}$ B.: Ciliary activity in the human and macaca nemestrina oviduct. Am J. Anat 138: 269, 1973.

20. McCOMB P F., HALBERT SA., GOMEL V: Pregnancy, ciliary transport, and the reversed ampullary segment of the rabbit fallopian tube. Fertil Steril 34: 386, 1980.

21. CROXATTO H B, ORTIZ M E., DIAZ S. HESS R., BALMACEDA J, CROXATTO H D.: Studies on the duration of egg transport by the human oviduct. II Ovum location at various interval following luteinizing hormone peak. Am J. Obstet Gynecol 132: 629,

22. TALO A, PULKKINEN M.: Electrical activity in the human oviduct during menstrual cycle. Am J. Obstet Gynecol 142: 135, 1982.

23. DONNEZ J., CASANAS-ROUX F, CAPRASSE J. FERRIN J. THOMAS K: Cycles changes in ciliation, cell height, and mitotic activity in human tubal epithelium during reproductive life. Fertil Steril 43: $554,1985$.

24. PULKKINEN O M., TALO A: Tubal physilogic consideration in ectopic pregnancy. Clin Obstet Gynecol 30: $164,1987$.

25. SIEGLER A.M.: Tubal plastic surgery; the past. the present and the future. Obstet Gynecol Surv 15: 680, 1960. 
26. PEARL R. SURFACE F.M.: Resection and end-to-end anatomosis of the oviduc in the hen without loss of function. Am J. Physiol 22:57, 1908.

27. CHRISTIAN S.L., SANDERSON E.L: A new method of anatomosing the ovarian tube or the vas deferens. JAMA 61: 2157, 1913.

28. SIEGLER A., HULKA J., PERETZ A: Reversibility of female sterilization. Fertil Steril 43: 499, 1985.

29. HOLDEN F C., SOVAK F.W.: Reconstruction of the oviducts: an improved technic with report of cases. Am J. Obstet Gynecol 24: 684, 1932.

30. PALMER R: Salpingostomy: a critical study of 396 personal cases operated upon without polyethylene tubing. Proc R. Soc Med. 53: 357, 1960.

31. SIEGLER A.M.: Salpingoplasty: Classification and report of 115 operations. Obstet Gynecol 34: 339, 1969.

32. ROCK J.A., TAKAYAMA K.P. MARTIN E.J., WOODRUFF J.D.,JONES HW J.R.: Factors influencing the success of salpingostomy technics for distal fimbrial obstruction. Obstet Gynecol 52: 591, 1978.

33. WINTON M.R.L.: Microsurgery of the fallopian tube: from fantasy to reality. Fertil Steril 34: 521, 1980.

34. GOMEL V: Clinical results of infertility microsurgery. in Female Infertility, Ed. by PG Crosignani, B.L. Rubin. London, Academic Press 1980 P. 77.

35. DeCHERNEY A.H., KASE N: A comparison of treatment for biletral fimbrial occlusión. Fertil Steril 35: 162, 1981.

36. KISTNER R.W., PATTON G.W.: Atlas of Infertility Surgery. Boston, Littl, Brown and Co, 1975.

37. PFEFFER W.H. Adjuvants in tubal surgery. Fertil Steril 33: 245, 1980.

38. JARRETT J., DAWOOD Y: Adhesion formation and uterine tube healing in the rabbit: A controlled study of the effects of ibuprofen and flurbiprofen. Am J. Obstet Gynecol 155: 1186, 1986.

39. DUNPHY J.E., UDUPA K.N.: Clinical and histochemical sequences in the normal healing of wounds. New Engl J. Med. 20: 847, 1955.

40. WEISSMANN G.: Prostanglandins in acute inflamation. Kalamazoo, Michigan: Scoe Publication. The Upjohn Co. 1: 29, 1980.

41. BATEMAN BG., NUNLEY WC., KITCHIN J.D.: Prevention of postoperative peritoneal adhesion with ibuprofen. Fertil steril 38: 107, 1982.

42. HOLTZ G: Faillure of a non steroidal anti-inflamatory agent (ibuprofen) to inhibit peritoneal adhesion reformation after lysis. Fertil Steril 37: 582, 1982.

43. O'BRIEN W.F., DRAKE T.S. BIBRO M.C.: The use of ibuprofen and dexamethasone in the prevention of postoperative adhesion formation. Obstet Gynecol 60: 373, 1982.

44. SIEGLER A., HULKA J. PERETZ A.: Reversibility of female sterilization. Fertil Steril 43: 499, 1985.
45. PHILLIPS J.M., HULKA B., KEITH 1, KEITH D: American association of Gynecologic Laparoscopist 1976 membership survey. J. Reprod Med. 2: 13,

46. ROCK J.A. GUZICK D.S., KATZ E., ZACUR H.A., KING T.M.: tubal anastomosis: pregnancy success following reversal of Falope ring or monopolar cautery sterilization Fertil Steril 48:13, 1987.

47. ROCK J.A., BERGQUIST C.A., ZACUR H.A., PARMLEY T.H., GUZICK D.S., JONES H.W., Jr: Tubal anatomosis following unipolar cautery. Fertil Steril 37: 611: 1982.

48. ROCK J.A., PARMLEY T. H., KING T.M., LAUFE L.E., Hsu B.C.: Endometriosis and the development of tuboperitoneal fistulas after tubal ligation. Fertil Steril 35: 16, 1981.

49. GOMEL V: Microsurgery in Female Infertility. Boston, Littl, Brown \& Co. 1983 p. 240.

50. Pelvic Infection: acute pelvic inflamatory disease. In Novak's Texbook of Gynecology, Eds Novak E.R., Jones G.S. Jones H.W. Baltimore. The Williams \& Wilkins Co. 1980 p. 348.

51. VASQUEZ G. WINSTON MRL, BOECKX W, GORDTS S. BROSENS IA: The epithelium of human hydrosalpinx: a light optical and scanning electron microscopic study BR. J. Obstet Gynecol 90: 764, 1983.

52. DONNEZ J. CASANAS-ROUX F: Prognostic factor of fimbrial microsurgery. Fertil Steril 42:200, 1986.

53. BATEMAN B., NUNLEY W., KITCHIN J: Surgical management of distal tubal obstruction are we making progress? Fertil Steril 48: 523, 1987.

54. BOER- MEISEL M.E., TE VELDE E.R., HABBEMA JDK, KARDAUM JWPF: Predicting the pregnancy outcome in patients treated for hydrosalpinx: a prospective study. Fertil Steril 45: 23, 1986.

55. TULANDI T. VILOS G.A.: A comparison between laser surgery and electrosurgery for bilateral hydrosalpinx: a 2 year follow-up. Fertil Steril 44: 846, 1985.

56. DANIELL J.F., DIAMOND M.P., McLAUGHLIN D.S., MARTIN D.C., FESTE J., SURRE MW., FRIEDMAN S., VAUGHN W.K: Clinical results of terminal salpingostomy with the use of the CO2 laser: report of the intraabdominal laser study group. Fertil Steril 45: 175, 1986.

57. FRANTZEN C., SCHLOSSER H-W: Microsurgery and postinfectious tubal infertility. Fertil Steril 38: 397, 1983.

58. PATTON G.W., Jr.: Pregnancy outcome following microsurgical fimbrioplasty. Fertil Steril 37: 150,

59. DONNEZ J. CASANAS - ROUX F: Prognostic factors of fimbrial microsurgery. Fertil Steril 46: 200, 1986.

60. KELLY R.W., ROBERTS D.K.: Experience with the carbon dioxide laser in gynecologic microsurgery. AM J. Obstet Gynecol 145: 585, 1983. 